\title{
MultiRBE: treatment planning for protons with selective radiobiological effectiveness
}

\author{
Daniel Sánchez-Parcerisaa $^{1,2 *}$, Miguel López-Aguirre ${ }^{1,2}$, Ana Dolcet Llerena ${ }^{3}$, José Manuel Udías ${ }^{1,2}$ \\ ${ }^{1}$ Grupo de Física Nuclear \& IPARCOS, Departamento de Estructura de la Materia, Física Térmica y \\ Electrónica, Universidad Complutense de Madrid, CEI Moncloa, 28040 Madrid, Spain.
}

${ }^{2}$ Instituto de Investigación Sanitaria del Hospital Clínico San Carlos (IdISSC), Madrid, Spain

${ }^{3}$ Qaelum NV, Leuven, Belgium

15

*Corresponding author: dsparcerisa@ucm.es

\begin{abstract}
Purpose: Clinical treatment planning protocols for protons recommend a uniform value radiobiological effectiveness (RBE) of protons of 1.1 throughout the treatment field, despite evidence from in-vitro and animal studies that proton RBE increases with linear energy transfer (LET), causing tissues placed distally to the target location to receive a presumably higher biological dose than estimated. While several voices in the medical physics community have advocated for variable RBE-based optimization, the uncertainties in RBE models have prevented its implementation in clinical practice, since an overestimation of RBE could cause significant target underdosage.
\end{abstract}

Methods: We propose a mixed RBE model (MultiRBE), where a uniform RBE is used in the target contours to ensure an adequate tumor coverage in terms of physical dose, but a variable RBE is used elsewhere. Our model was implemented in the open-source treatment planning system matRad and three example cases were planned: a homogeneous phantom, a prostate tumor and a head-andneck case. MultiRBE was used for plan optimization, and the produced plans were subsequently evaluated in terms of physical dose coverage $\left(\mathrm{V}_{95 \%}\right)$ and variable RBE-weighted dose in organs at risk and normal tissue complication probabilities (NTCP), where prediction models were available.

Results: The planning algorithm showed potential for reducing the biological dose in organs surrounding the planning target and thus decreasing the probability for complications in normal tissue (by up to $62 \%$ in the prostate case and $37 \%$ in the head-and-neck patient). This was achieved without compromising the target coverage or homogeneity in terms of physical dose, as a result of a smarter redistribution of dose among the surrounding tissues with regard to the optimization constraints.

Conclusions: The results prove the ability of the MultiRBE model to reduce biological dose at healthy tissues without compromising the dose coverage of the tumor, with independence of the variable RBE models used.

\section{Introduction}

Clinical protocols for proton radiotherapy recommend the use of a relative biological effectiveness (RBE) uniform and equal to 1.1 across the treatment field [1], in contrast to what is common in carbon ion radiotherapy, where variable RBE models are incorporated in clinical practice [1]. The rationale for this recommendation is complex: while proton RBE has shown variations as a function of biological endpoint, dose, and linear energy transfer in in-vivo and in-vitro experiments [2], proton RBE values based on humanresponse are scarce. Therefore, a consensus was reached to adopt a generic value of 1.1 for proton RBE, but, at the same time, to consider the range uncertainty originating from increased RBE in the distal edge of proton 
fields [3] by not pointing proton fields at organs at risk situated directly behind the target, or by lowering certain dose constraints.

Some clinical groups have investigated possible correlations between increased proton LET values and occurrence of normal tissue complications in patients treated with proton therapy [10][11], and, while some effect was indeed observed, they have failed to establish statistical significance, partly due to the reduced number of cases reported. Other case reports on toxicities in pediatric patients called for attention to potential effects of increased RBE: Merchant [12] demanded caution in the treatment of pediatric patients, particularly on ependymoma, due to late reactions in the brainstem, and Indelicato et al suggested more conservative dosimetric guidelines in young patients with posterior fossa tumors [13].

10 While the evidence supporting variable proton RBE due to increased LET in in-vitro and animal studies is solid [5][6][7][69], the first reports of statistically significant data in human subjects (although using subclinical endpoints) were published only recently. A study in 34 ependymoma patients found a correlation between incidence of post-treatment image changes in magnetic resonance and both dose and LET [8]. More recently, a similar study in chest wall patients reported a correlation between asymptomatic late-phase radiographic changes within the lung and increased proton RBE [9]. These new findings (along with accumulated clinical experience) have triggered several reviews of the existing knowledge on proton RBE. While a few of them claim, in one way or another, that a variable RBE scheme should be implemented in clinical practice in the near future [14][15][16] many others agree that current evidence still does not support such a change of paradigm and propose alternative solutions for the consideration of this effect [17][18][19][20][21][22][23]. In fact, the conclusions of a recent 'expert group' [24] explicitly mention that "the clinical practice of using a fixed RBE of 1.1 cannot be abandoned based on high-quality evidence favoring other values in specific situations", although they call for further research as the increasing capability to accurately deliver the dose in the treatment will cause the proton Bragg peaks to be positioned more precisely at the same spot, resulting in repeated exposure of areas with elevated LET values.

Several strategies have been proposed in the past to tackle the variable RBE problem in proton radiotherapy, either using empirical models based on in-vitro survival data [25][26][27][28] or in more complex, physical models such as the local effect model [29][30] or the micro-kinetic model [31][32]. For example, Frese et al proposed a biological optimization system for radioresistant tumors [33]. These strategies often face criticism for possible underdosage of the target due to uncertainties in RBE. Besides, a recent study in prostate cancer [34] has found that it is not possible to simultaneously meet the tumor and rectal constrains for both physical and variable RBE-weighted dose, the latter being estimated based on in vitro cell survival data. In fact, other groups have decided to avoid the sometimes controversial concept of proton RBE completely and have tackled this problem by performing the optimization directly on LET distributions [35][36][37][38][39] or proposing new beam orientations or treatment modalities [40][41][42].

Our proposed solution is the use of a mixed RBE model (MultiRBE) for plan optimization, where a uniform RBE is used in the target contours to ensure an adequate tumor coverage in terms of physical dose, but a variable RBE is used elsewhere. This solution incorporates the benefits of both approaches: it produces a quantifiable, numeric RBE quantity that can be used for weighting of physical dose, but it also ensures appropriate coverage of the target with a flat physical dose distribution.

\section{Materials and methods}

\subsection{Implementation}

The MultiRBE planning strategy was implemented in the non-commercial treatment planning system matRad [43], developed on an open-source basis as a MATLAB library which supports inverse planning for IMRT and actively scanned protons and carbon ion beams. Dose calculation in matRad is carried out using modified pencil-beam algorithms [44][45] and a set of Monte Carlo-calculated physical base data [46]. Unlike other treatment planning systems with a similar purpose, such as $\mathrm{FoCa}$ [47], proton LET calculation (required for RBE estimation) is not derived from analytical models [48], but pre-loaded from a Monte Carlo base data and 
transported across the voxelized geometry.

The example treatment machine provided with matRad was used to produce all plans in this study. Therefore, the findings here reported are non-specific to any particular device; concrete limitations of each particular machine (available ranges or treatment angles, for example) might limit or modulate the conclusions drawn from our study and should be analyzed on a case-by-case basis.

\subsection{MultiRBE model}

10 Proton RBE, defined as the ratio between photon reference dose $\left(D_{x}\right)$ and proton dose $\left(D_{p}\right)$ at isoeffect, can be modeled using the linear quadratic (LQ) model, where the survival fraction (SF) of cells in a clonogenic assay is expressed as a function of dose in $S F=\exp \left(-\alpha D-\beta D^{2}\right)$. Using the $\mathrm{RBE}_{\min }$ and $\mathrm{RBE}_{\max }$ concepts established by Carabe et al [26] and the in-vitro cell survival datasets compiled by Paganetti [3], McNamara and colleagues [28] derived the following expression for proton RBE, which depends only on proton dose per

15 fraction and dose-averaged LET, as well as on the $\alpha / \beta$ parameter measured with photon reference radiation, denoted $\left(\frac{\alpha}{\beta}\right)_{x}$ :

$R B E\left[D_{p},\left(\frac{\alpha}{\beta}\right)_{x}, L E T\right]=\frac{1}{2 D_{p}}\left(\sqrt{\left(\frac{\alpha}{\beta}\right)_{x}^{2}+4 D_{p}\left(\frac{\alpha}{\beta}\right)_{x}\left(p_{0}+\frac{p_{1}}{\left(\frac{\alpha}{\beta}\right)_{x}} L E T\right)+4 D_{p}^{2}\left(p_{2}+p_{3} \sqrt{\left(\frac{\alpha}{\beta}\right)_{x}} L E T\right)^{2}}-\left(\frac{\alpha}{\beta}\right)_{x}\right)$

20 and the derived values for the fitting parameters are $\mathrm{p}_{0}=0.99 \pm 0.01, \mathrm{p}_{1}=0.36 \pm 0.02 \mathrm{keV} / \mu \mathrm{m} \cdot \mathrm{Gy}^{-1}$, $\mathrm{p}_{2}=1.101 \pm 0.006$ and $\mathrm{p}_{3}=-0.0039 \pm 0.0006(\mathrm{keV} / \mu \mathrm{m})^{-1} \cdot \mathrm{Gy}^{-1 / 2}$.

In our implementation of MultiRBE, we plan with McNamara's model of RBE throughout the complete irradiation field, except for the planning target volumes (PTVs). In these volumes, we use a uniform value of $\mathrm{RBE}=1.1$. We chose the McNamara model for variable RBE (over other models such as Carabe [26] or Wedenberg [27]) as it is, from all the empirical models, the one which is derived from the largest and most varied dataset.

\subsection{Patient selection and choice of alpha/beta values}

Three example cases were chosen for the tests of the MultiRBE model: a C-shaped target drawn in a homogeneous water phantom (described in the TG-119 report [49]), a prostate cancer, and a head and neck carcinoma. They belong to the CORT DataSet [50], a set of shared data designed for IMRT treatment planning research.

In order to calculate RBE values using the McNamara model, alpha/beta values were required for all volumes of interest. These were extracted from consensus values available from the literature or important reference papers and are detailed in Table 1. In the case of the homogeneous water phantom, arbitrary values were chosen. It is outside the scope of this article to discuss on an optimal choice of alpha/beta ratios: the values here presented are quoted for reference purposes only and, should the MultiRBE concept be incorporated in clinical protocols, the alpha/beta values could have to be reevaluated based on the judgement of the oncology team.

Table 1. Choice of alpha/beta values in tumor and healthy tissues.

\begin{tabular}{|l|c|l|}
\hline Tissue & Alpha/beta [Gy] & Source \\
\hline Phantom - body and core & 3.0 & - \\
\hline Phantom - tumor & 10.0 & - \\
\hline Prostate tumor & 1.4 & Miralbell et al, 2012 [51] \\
\hline Rectum & 3.9 & Emami et al, 1991 [52] \\
\hline Bladder & 6.0 & Emami et al, 1991 [52] \\
\hline
\end{tabular}




\begin{tabular}{|l|c|c|}
\hline Femoral heads & 0.9 & Emami et al, 1991 [52] \\
\hline Head and neck carcinoma & 10.5 & Stuschke \& Thames, 1999 [53] \\
\hline Larynx & 4.2 & Emami et al, $1991[52]$ \\
\hline Parotid glands & 3.0 & Emami et al, 1991 [52] \\
\hline Spinal cord & 3.3 & Emami et al, 1991 [52] \\
\hline Arbitrary / muscle-vascular & 3.1 & Withers et al, $1995[54]$ \\
\hline
\end{tabular}

\subsection{Study design, optimization constraints and evaluation metrics}

We designed a dosimetric study comparing the MultiRBE planning algorithm with the standard planning strategy $(\mathrm{RBE}=1.1)$ and the use of a variable RBE (based on the McNamara model) throughout the complete treatment field.

For each of the three analyzed cases, fixed optimization objectives (with fixed relative importance factors, shown in Table 2) were defined and used to create three different IMPT (allowing for multi-field optimization) plans (MultiRBE, variable RBE and uniform RBE). In order to ensure a fair assessment of the models, no further human intervention was applied to any of the plans after applying the optimization objectives. A full description of the optimization process and cost functions is described in the reference paper for matRad [43]. On a second stage (see scheme in Figure 1), each of these plans re-evaluated (RBE-weighted doses recalculated) in terms of uniform and variable RBE models.

Table 2a. Optimization objectives for case 1: homogeneous phantom

\begin{tabular}{|l|l|l|}
\hline Region of interest & Objective & Penalty \\
\hline Inner core & $\begin{array}{l}5 \% \text { of the volume receiving } \\
\text { less than 10 GyRBE }\end{array}$ & 1000 \\
\hline Target & $\begin{array}{l}\text { Square deviation of objective } \\
\text { dose of 50 GyRBE }\end{array}$ & 1000 \\
\cline { 2 - 3 } $\begin{array}{l}\text { 99\% of the volume receiving } \\
\text { at least 50 GyRBE }\end{array}$ & 3000 \\
\cline { 2 - 3 } & $\begin{array}{l}10 \% \text { of the volume receiving } \\
\text { not more than 55 GyRBE }\end{array}$ & 500 \\
\hline Body - Target & $\begin{array}{l}\text { Square overdosing for doses } \\
\text { above 30 GyRBE }\end{array}$ & 100 \\
\hline
\end{tabular}

Table 2b. Optimization objectives for case 2: prostate

\begin{tabular}{|l|l|l|}
\hline Region of interest & Objective & Penalty \\
\hline Target & $\begin{array}{l}\text { Square deviation of objective } \\
\text { dose of 78 GyRBE }\end{array}$ & 1000 \\
\hline Bladder & $\begin{array}{l}5 \% \text { of the volume receiving } \\
\text { not more than 70 GyRBE }\end{array}$ & 500 \\
\cline { 2 - 3 } & $\begin{array}{l}\text { 20\% of the volume receiving } \\
\text { not more than 40 GyRBE }\end{array}$ & 500 \\
\hline Rectum & $\begin{array}{l}5 \% \text { of the volume receiving } \\
\text { not more than 70 GyRBE }\end{array}$ & 2500 \\
\hline Femoral heads (each) & $\begin{array}{l}5 \% \text { of the volume receiving } \\
\text { not more than 50 GyRBE }\end{array}$ & 500 \\
\hline Body - Target & $\begin{array}{l}\text { Square overdosing for doses } \\
\text { above 30 GyRBE }\end{array}$ & 100 \\
\hline
\end{tabular}


Table 2c. Optimization objectives for case 3: head and neck

\begin{tabular}{|l|l|l|}
\hline Region of interest & Objective & Penalty \\
\hline PTV70 & $\begin{array}{l}\text { Square deviation of objective } \\
\text { dose of 70 GyRBE }\end{array}$ & 1000 \\
\hline PTV63 & $\begin{array}{l}\text { Square deviation of objective } \\
\text { dose of 63 GyRBE }\end{array}$ & 1000 \\
\hline Brainstem & $\begin{array}{l}1 \% \text { of the volume receiving } \\
\text { not more than 54 GyRBE }\end{array}$ & 100 \\
\hline Optic Chiasm & $\begin{array}{l}1 \% \text { of the volume receiving } \\
\text { not more than 54 GyRBE }\end{array}$ & 100 \\
\hline Larynx & $\begin{array}{l}\text { Square overdosing for doses } \\
\text { above 40 GyRBE }\end{array}$ & 100 \\
\hline Parotids (each) & $\begin{array}{l}\text { Square overdosing for doses } \\
\text { above 20 GyRBE }\end{array}$ & 500 \\
\hline Spinal cord & $\begin{array}{l}1 \% \text { of the volume receiving } \\
\text { not more than 50 GyRBE }\end{array}$ & 100 \\
\hline Temporal lobes (each) & $\begin{array}{l}1 \% \text { of the volume receiving } \\
\text { not more than 60 GyRBE }\end{array}$ & 100 \\
\hline Body - Target & $\begin{array}{l}\text { Square overdosing for doses } \\
\text { above 30 GyRBE }\end{array}$ & 800 \\
\hline & & \\
\hline
\end{tabular}

Plans were evaluated in terms of the resulting dose-volume histograms (DVH). To investigate the effect of the choice of RBE model in dose redistribution among the different OAR (rather than on overall total dose), DVHs were calculated after re-normalizing the physical dose so that the mean biological dose in the target equaled the prescription, using an RBE of 1.1 . Dose covering $95 \%$ of the target volume, or $\mathrm{D}_{95}$, was selected as a coverage metric for PTV, and OAR overdosage was assessed using site-specific NTCP models. A conservative approach was used to evaluate these biological dose distributions: all metrics were evaluated in terms of both fixed-RBE (expressed in GyRBE ${ }_{1.1}$ ) and variable-RBE (expressed in $\mathrm{GyRBE}_{\mathrm{McN}}$ ) weighted physical doses, so that the utility of the MultiRBE concept can be compared to both alternatives. This information is displayed in tables 3-5, extracted before and after normalization of the total dose.

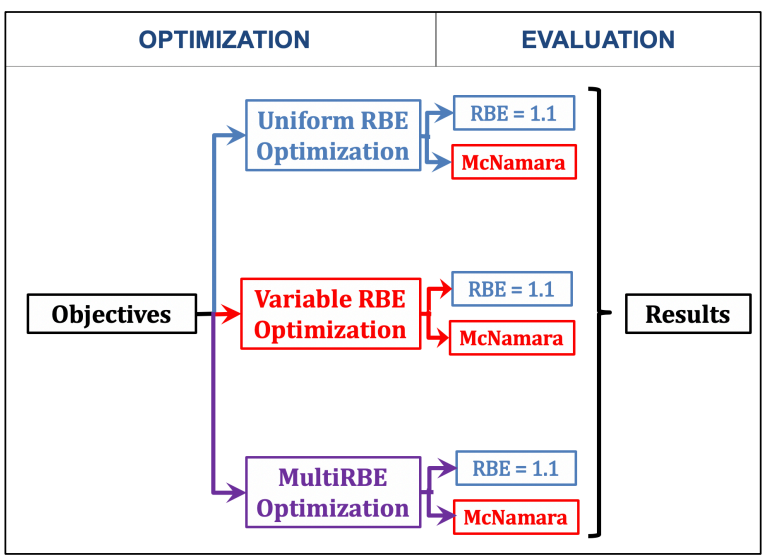

Figure 1. Scheme of the procedure for the study 


\section{Results}

\subsection{Homogeneous phantom}

5 A C-shaped target in a homogeneous water phantom was prescribed with 50 GyRBE to the target volume (distributed across 30 fractions) and a maximum of 10 GyRBE to 5\% of the core volume [49], a cylindrical shape situated within the target. In order to maximize biological effect, a single anterior proton field was used to plan the case using the three planning strategies. Figure 2 and Table 3 show, respectively, the DVH and dose statistics for the three analyzed RBE models.

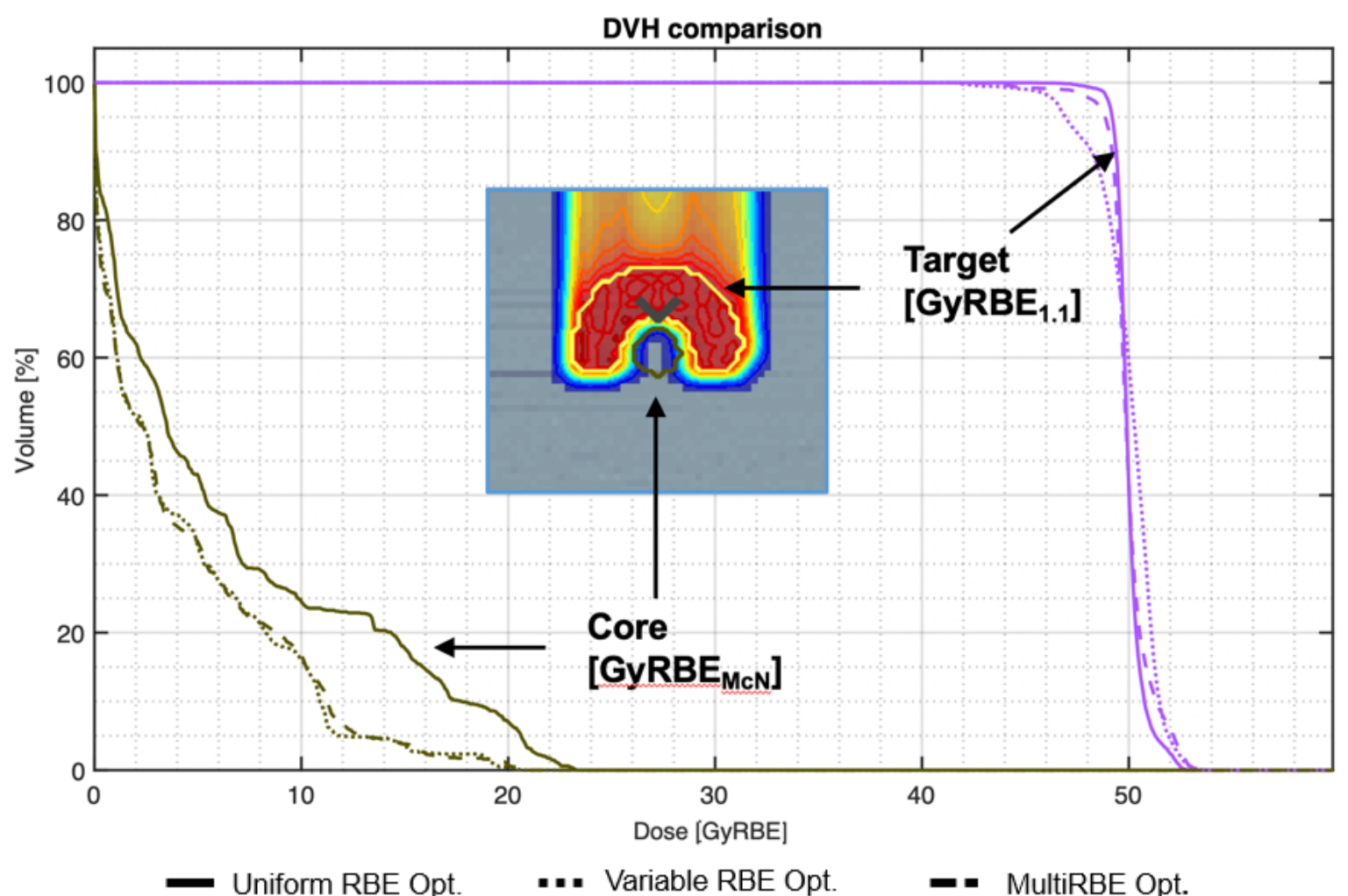

Figure 2. Dose-volume histogram for TG-119 phantom plan, after normalization of target dose to 50 $G y R B E_{1.1}$. Note that $O A R$ doses are expressed in GyRBE $E_{M c N}$, while target doses are expressed in GyRBE $E_{1.1}$. Inlet: $2 D$ slice of RBE-weighted dose in target.

Table 3. Metrics for three planning strategies for the phantom case, before and after normalization of target

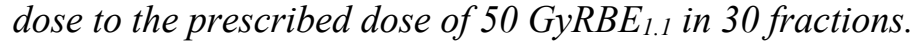

\begin{tabular}{|l|c|c|c|c|c|c|}
\hline & \multicolumn{3}{|c|}{ Before normalization } & \multicolumn{3}{c|}{ After normalization } \\
\hline Metric & $\begin{array}{c}\text { Uniform } \\
\text { RBE }\end{array}$ & $\begin{array}{c}\text { Variable } \\
\text { RBE }\end{array}$ & $\begin{array}{c}\text { Multi } \\
\text { RBE }\end{array}$ & $\begin{array}{c}\text { Uniform } \\
\text { RBE }\end{array}$ & $\begin{array}{c}\text { Variable } \\
\text { RBE }\end{array}$ & $\begin{array}{c}\text { Multi } \\
\text { RBE }\end{array}$ \\
\hline Target $\mathrm{D}_{95}\left[\mathrm{GyRBE}_{1.1}\right]$ & 49.4 & 47.1 & 49.7 & 49.2 & 46.9 & 48.9 \\
\hline Target $\mathrm{D}_{95}\left[\mathbf{G y R B E}_{\mathrm{McN}}\right]$ & 48.6 & 49.3 & 49.1 & 48.4 & 49.1 & 48.2 \\
\hline Core $\mathrm{D}_{5}\left[\mathrm{GyRBE}_{1.1}\right]$ & 11.3 & 8.3 & 8.6 & 11.2 & 8.3 & 8.4 \\
\hline Core $\mathrm{D}_{5}\left[\mathrm{GyRBE}_{\mathrm{McN}}\right]$ & 20.7 & 11.8 & 13.0 & 20.6 & 11.7 & 12.8 \\
\hline
\end{tabular}

20 This case is a clear example of radiobiological range uncertainty, because the OAR is situated directly distal to the target and therefore receives higher average LET. In this example, a variable RBE strategy reduces the biological dose received by $5 \%$ of the core volume $\left(\right.$ Core $\left.\mathrm{D}_{5}\right)$ by $43 \%$, but it fails to maintain target coverage in terms of physical dose. On the contrary, the suggested MultiRBE planning strategy achieves a reduction of $37 \%$ in Core $\mathrm{D}_{5}$ without compromising the $\mathrm{D}_{95}$ at the PTV (in fact, target coverage $\mathrm{D}_{95}$ is slightly better at the cost of a slightly decreased target homogeneity). For this geometry, $\mathrm{RBE}_{\mathrm{McN}}$ in the target takes values both 
above and below 1.1, so depending on the optimized plans, mean $\mathrm{RBE}_{\mathrm{McN}}$ can be lower than 1.1 (as is the case for uniform RBE and MultiRBE optimized plans) or higher than 1.1 (as in the variable-RBE optimized plan).

\subsection{Prostate tumor}

A prostate tumor was planned with standard proton plan with two opposed lateral beams. The target volume was prescribed with 78 GyRBE in 39 fractions. Restrictions of $\mathrm{V}_{70 \mathrm{~Gy}}<30 \%$ and $\mathrm{V}_{50 \mathrm{~Gy}}<50 \%$ were applied to the rectum, $\mathrm{V}_{70 \mathrm{~Gy}}<35 \%$ and $\mathrm{V}_{50 \mathrm{~Gy}}<60 \%$ to the bladder and $\mathrm{V}_{50 \mathrm{~Gy}}<5 \%$ to the femoral heads [68]. Figure 3 and Table 4 (left) show, respectively, the dose-volume histogram (DVH) and dose statistics for the three analyzed RBE models. Due to the increased $\mathrm{RBE}_{\mathrm{McN}}$ in the target volume (with an average value of 1.26 within the PTV), the plan constructed with variable RBE produced, for a given prescription of $78 \mathrm{GyRBE}$, an overall lower dose throughout the complete treatment volume. The resulting DVHs and dose metrics are shown in Figure 3 and Table 4 . Note how for both $\mathrm{RBE}_{1.1}$ and $\mathrm{RBE}_{\mathrm{McN}}$ models independently, both bladder and rectum maximum doses are below the maximum target dose, and in all cases below $107 \%$ of the standard prescription dose of $78 \mathrm{GyRBE}_{1.1}$. The probability of rectal bleeding was derived from the dose distributions using four different NTCP models [55-58]. In this case, the MultiRBE model yields an average reduction of $37 \%$ in the probability of rectal bleeding according to four different models [min $22 \%$ - max $62 \%$ ] compared to the standard RBE-weighted dose, with no relevant effect in target coverage.

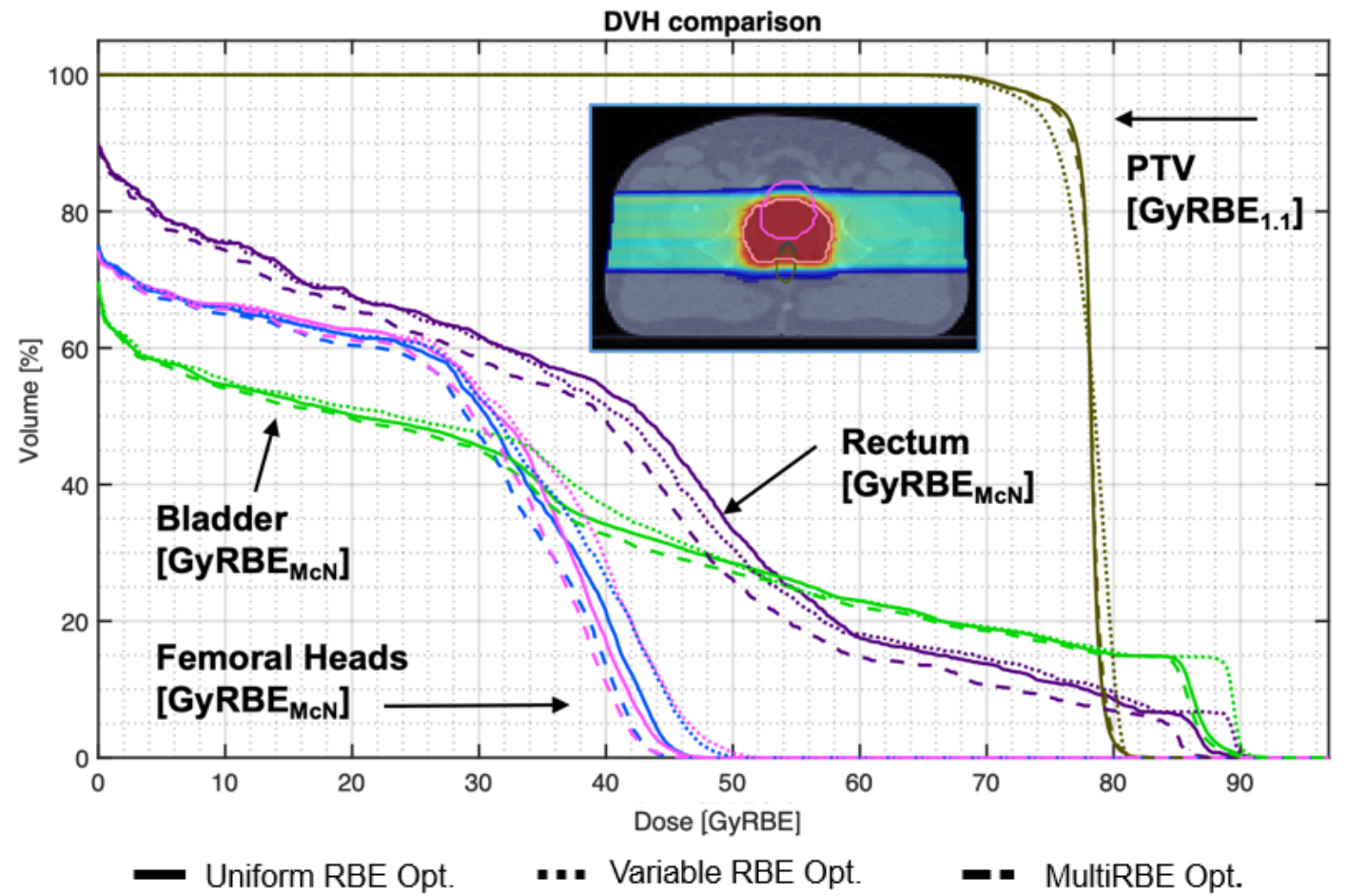

Figure 3. Dose-volume histogram for prostate plans, after normalization of target dose to the prescribed dose of $78 G y R B E_{1.1}$. Note that OAR doses are expressed in GyRBE $E_{M c N}$, while target doses are expressed in GyRBE $E_{1.1}$. Inlet: $2 D$ slice of RBE-weighted dose.

Table 4. Metrics for three planning strategies for the prostate case, before and after normalization of target dose to the prescribed dose of $78 G y R B E_{1.1}$ in 39 fractions, including normal-tissue complication probabilities.

\begin{tabular}{|c|c|c|c|c|c|c|}
\hline & \multicolumn{3}{|c|}{ Before normalization } & \multicolumn{3}{|c|}{ After normalization } \\
\hline Metric & $\begin{array}{c}\text { Uniform } \\
\text { RBE }\end{array}$ & $\begin{array}{c}\text { Variable } \\
\text { RBE }\end{array}$ & MultiRBE & $\begin{array}{c}\text { Uniform } \\
\text { RBE }\end{array}$ & $\begin{array}{c}\text { Variable } \\
\text { RBE }\end{array}$ & MultiRBE \\
\hline Target $\mathrm{D}_{95}\left[\mathrm{GyRBE}_{1.1}\right]$ & 75.5 & 64.4 & 77.7 & 75.8 & 73.9 & 75.4 \\
\hline Target $\mathrm{D}_{95}\left[\mathrm{GyRBE}_{\mathrm{McN}}\right.$ ] & 84.6 & 75.8 & 86.7 & 84.9 & 87.0 & 84.0 \\
\hline Rectum V70Gy $\left[\mathrm{GyRBE}_{\mathrm{McN}}\right.$ ] & $13.7 \%$ & $8.9 \%$ & $12.1 \%$ & $13.8 \%$ & $14.5 \%$ & $10.6 \%$ \\
\hline Rectum V70Gy $\left[\mathrm{GyRBE}_{1.1}\right]$ & $11.6 \%$ & $0.1 \%$ & $10.7 \%$ & $11.6 \%$ & $12.3 \%$ & $10.1 \%$ \\
\hline
\end{tabular}




\begin{tabular}{|c|c|c|c|c|c|c|}
\hline Bladder $\mathrm{V}_{70 \mathrm{~Gy}}\left[\mathrm{GyRBE}_{\mathrm{McN}}\right]$ & $18.9 \%$ & $15.3 \%$ & $19.1 \%$ & $18.9 \%$ & $19.1 \%$ & $18.6 \%$ \\
\hline Bladder $V_{70 \mathrm{~Gy}}\left[\mathrm{GyRBE}_{1.1}\right]$ & $18.4 \%$ & $2.2 \%$ & $18.6 \%$ & $18.5 \%$ & $18.3 \%$ & $18.0 \%$ \\
\hline Target max dose [GyRBE $\left.{ }_{1.1}\right]$ & 82.5 & 70.9 & 85.9 & 82.9 & 81.3 & 83.3 \\
\hline Target max dose [GyRBE $\mathrm{McN}]$ & 95.6 & 83.4 & 99.0 & 96.0 & 95.6 & 96.0 \\
\hline Rectum max dose [GyRBE 1.1$]$ & 79.4 & 70.3 & 82.8 & 79.7 & 80.6 & 80.3 \\
\hline Rectum max dose [GyRBEMcN] & 90.1 & 79.1 & 90.8 & 90.4 & 90.8 & 88.0 \\
\hline Bladder max dose [GyRBE $\left.{ }_{1.1}\right]$ & 80.2 & 70.9 & 83.3 & 80.5 & 81.3 & 80.7 \\
\hline Bladder maxdose [GyRBE $\left.{ }_{M c N}\right]$ & 91.9 & 80.6 & 94.6 & 92.2 & 92.4 & 91.7 \\
\hline $\begin{array}{l}\text { Prob. of rectal bleeding [55] } \\
\text { [calculated from GyRBEMcN] }\end{array}$ & $11.8 \%$ & $4.0 \%$ & $8.7 \%$ & $12.2 \%$ & $15.3 \%$ & $8.7 \%$ \\
\hline $\begin{array}{l}\text { Prob. of rectal bleeding [55] } \\
\text { [calculated from GyRBE }\end{array}$ & $5.2 \%$ & $1.3 \%$ & $6.1 \%$ & $5.3 \%$ & $5.6 \%$ & $4.4 \%$ \\
\hline $\begin{array}{l}\text { Prob. of rectal bleeding [56] } \\
\text { [calculated from GyRBE }\end{array}$ & $26.6 \%$ & $9.9 \%$ & $21.2 \%$ & $27.2 \%$ & $33.5 \%$ & $21.2 \%$ \\
\hline $\begin{array}{l}\text { Prob. of rectal bleeding [56] } \\
\text { [calculated from GyRBE } 1.1]\end{array}$ & $11.7 \%$ & $2.9 \%$ & $14.6 \%$ & $12.1 \%$ & $13.0 \%$ & $10.8 \%$ \\
\hline $\begin{array}{l}\text { Prob. of rectal bleeding [57] } \\
\text { [calculated from GyRBE }\end{array}$ & $7.5 \%$ & $2.1 \%$ & $5.1 \%$ & $7.8 \%$ & $10.0 \%$ & $5.1 \%$ \\
\hline $\begin{array}{l}\text { Prob. of rectal bleeding [57] } \\
\text { [calculated from GyRBE }\end{array}$ & $2.9 \%$ & $0.6 \%$ & $3.4 \%$ & $3.0 \%$ & $3.2 \%$ & $2.3 \%$ \\
\hline $\begin{array}{l}\text { Prob. of rectal bleeding [58] } \\
\text { [calculated from GyRBEMcN] }\end{array}$ & $7.4 \%$ & $1.7 \%$ & $2.9 \%$ & $7.6 \%$ & $9.4 \%$ & $2.9 \%$ \\
\hline $\begin{array}{l}\text { Prob. of rectal bleeding [58] } \\
\text { [calculated from GyRBE1.1] }\end{array}$ & $4.0 \%$ & $0.1 \%$ & $3.0 \%$ & $4.0 \%$ & $4.9 \%$ & $2.5 \%$ \\
\hline
\end{tabular}

\subsection{Head and neck carcinoma}

Finally, we planned a head \& neck carcinoma with two lateral oblique beams at $+60^{\circ}$ and $-60^{\circ}$ gantry angles.

Two target volumes were prescribed with 70 GyRBE and 63 GyRBE in 35 fractions, mean doses at the larynx and parotids were restricted to $40 \mathrm{~Gy}$ and 20 Gy respectively, while maximum doses were limited at serial organs: cord (50 Gy), brainstem (54 Gy), optic nerves and chiasm (54 Gy), temporal lobes (60 Gy) and eye ball (25 Gy) [66]. Figure 4 and Table 5 show the dose-volume histogram (DVH) and dose statistics for the three analyzed RBE models.

This case is different to the other shown since the McNamara model predicts an average RBE of 1.08 (lower than 1.1) for the PTV. This is caused by a combination of a high alpha/beta value for the tumor and a rather large modulation of the spread-out Bragg peak (up to $12 \mathrm{~cm}$ ), causing the PTV to be irradiated with lowerLET parts of the proton field: according to Eq. 1, for a dose per fraction of 2 Gy and an alpha/beta value of $10.5 \mathrm{~Gy}$ in the target, only radiation with an LET above $3.7 \mathrm{keV} / \mu \mathrm{m}$ (which is found only at the most distal $\mathrm{mm}$ of the depth dose distribution [48]) has an RBE above 1.1. Therefore, the physical dose in the target obtained with variable RBE optimization is larger than with the constant or MultiRBE models.

Accounting for variable RBE in the planning process produces a redistribution of the dose in the organs at risk, moving dose from the parotids to organs where constraints are easier to meet (larynx, spinal cord). The MultiRBE planning algorithm achieves a reduction of $7 \%$ in the probability of xerostomia [61] and $37 \%$ in the probability of parotid fibrosis [62], compared to the standard RBE-weighted dose, without detriment in target coverage or homogeneity. 


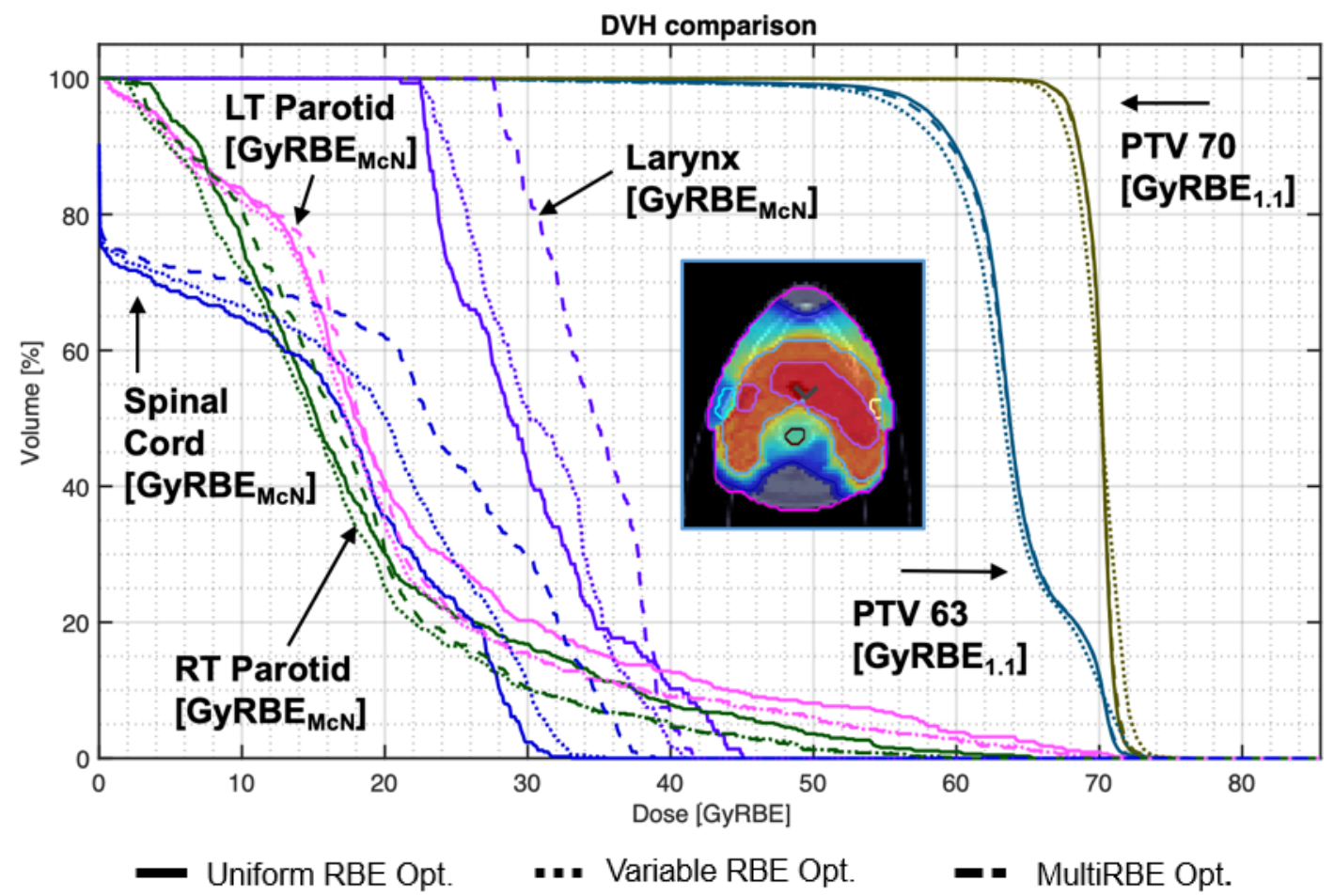

Figure 4. Dose-volume histogram for head and neck plans, after normalization of PTV70 dose to the prescribed dose of $70 G y R B E_{1.1}$ in 35 fractions. Note that OAR doses are expressed in $G y R B E_{M c N}$, while target doses are expressed in GyRBE $E_{1.1}$. Inlet: $2 D$ slice of RBE-weighted dose.

Table 5. Metrics for three planning strategies for the head and neck case, before and after normal-

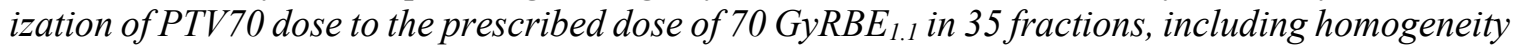
indexes (HI) and normal-tissue complication probabilities.

\begin{tabular}{|c|c|c|c|c|c|c|}
\hline \multirow[b]{2}{*}{ Metric } & \multicolumn{3}{|c|}{ Before normalization } & \multicolumn{3}{|c|}{ After normalization } \\
\hline & $\begin{array}{l}\text { Uniform } \\
\text { RBE }\end{array}$ & $\begin{array}{l}\text { Variable } \\
\text { RBE }\end{array}$ & $\begin{array}{l}\text { Multi } \\
\text { RBE }\end{array}$ & $\begin{array}{l}\text { Uniform } \\
\text { RBE }\end{array}$ & $\begin{array}{l}\text { Variable } \\
\text { RBE }\end{array}$ & $\begin{array}{l}\text { Multi } \\
\text { RBE }\end{array}$ \\
\hline PTV70 D $95\left[\mathrm{GyRBE}_{1.1}\right]$ & 69.7 & 68.5 & 68.6 & 68.0 & 67.2 & 67.9 \\
\hline PTV70 D $95\left[\mathrm{GyRBE}_{\mathrm{McN}}\right]$ & 68.0 & 67.8 & 66.7 & 66.1 & 66.6 & 66.1 \\
\hline $\begin{array}{l}\mathrm{HI}=\mathrm{D}_{2} / \mathrm{D}_{98} \text { at PTV70 } \\
\left.\text { [GyRBE }_{1.1}\right]\end{array}$ & 1.07 & 1.10 & 1.07 & 1.07 & 1.10 & 1.07 \\
\hline $\begin{array}{l}\mathrm{HI}=\mathrm{D}_{2} / \mathrm{D}_{98} \text { at PTV70 } \\
{\left[\mathrm{GyRBE}_{\mathrm{McN}}\right]}\end{array}$ & 1.09 & 1.07 & 1.09 & 1.09 & 1.07 & 1.09 \\
\hline $\mathrm{D}_{\text {mean }}$ left parotid [GyRBE 1.1$]$ & 20.7 & 19.4 & 20.0 & 20.8 & 19.1 & 19.8 \\
\hline $\begin{array}{l}\text { Dmean left parotid } \\
{\left[\mathrm{GyRBE}_{\mathrm{McN}}\right]}\end{array}$ & 22.0 & 20.4 & 20.9 & 22.1 & 20.0 & 20.7 \\
\hline $\begin{array}{l}\mathrm{D}_{\text {mean }} \text { right parotid } \\
{\left[\mathrm{GyRBE}_{1.1}\right]}\end{array}$ & 17.4 & 16.0 & 17.3 & 17.5 & 15.8 & 17.1 \\
\hline $\begin{array}{l}D_{\text {mean }} \text { right parotid } \\
{\left[\text { GyRBE }_{\mathrm{McN}}\right]}\end{array}$ & 18.6 & 16.9 & 17.9 & 18.7 & 16.5 & 17.7 \\
\hline $\begin{array}{l}\text { Probability of xerostomia } \\
{\left[\text { GyRBE }_{1.1}\right][59]}\end{array}$ & $20.1 \%$ & $17.8 \%$ & $19.8 \%$ & $20.2 \%$ & $17.4 \%$ & $19.6 \%$ \\
\hline $\begin{array}{l}\text { Probability of xerostomia } \\
\text { [GyRBEMcN] [59] }\end{array}$ & $22.1 \%$ & $19.1 \%$ & $20.9 \%$ & $22.2 \%$ & $18.6 \%$ & $20.6 \%$ \\
\hline $\begin{array}{l}\text { Probability of fibrosis (left } \\
\text { parotid) [GyRBE } 1.1][60]\end{array}$ & $6.5 \%$ & $4.0 \%$ & $5.0 \%$ & $6.7 \%$ & $3.4 \%$ & $4.7 \%$ \\
\hline
\end{tabular}




\begin{tabular}{|l|c|c|c|c|c|c|}
\hline $\begin{array}{l}\text { Probability of fibrosis (left } \\
\text { parotid) [GyRBE }\end{array}$ & $10.5 \%$ & $5.9 \%$ & $7.1 \%$ & $10.8 \%$ & $5.1 \%$ & $6.6 \%$ \\
\hline $\begin{array}{l}\text { Probability of fibrosis (right } \\
\text { parotid) [GyRBE }\end{array}$ & $1.6 \%$ & $0.8 \%$ & $1.5 \%$ & $1.7 \%$ & $0.7 \%$ & $1.4 \%$ \\
\hline $\begin{array}{l}\text { Probability of fibrosis (right } \\
\text { parotid) [GyRBE }\end{array}$ & $2.7 \%$ & $1.2 \%$ & $2.0 \%$ & $2.8 \%$ & $1.0 \%$ & $1.9 \%$ \\
\hline
\end{tabular}

\section{Discussion and conclusions}

The proposed MultiRBE planning algorithm has shown a clear potential for reducing the biological dose in organs surrounding the planning target and thus decreasing the probability for complications in normal tissues, by around $30 \%$ (between $11 \%$ and $62 \%$, depending on the models) in the prostate case and around $20 \%$ (between 3\% and 38\%) in the head and neck patient. This is achieved without compromising the target coverage or homogeneity in terms of physical dose, as a result of a smarter redistribution of dose among the surrounding tissues with regard to the optimization constraints. Moreover, the algorithm could be introduced in the clinical workflow, requiring only of an additional step (inclusion of the alpha/beta values) in the treatment planning process, provided that the TPS supports RBE and LET calculation [48].

The MultiRBE idea is not entirely new: in fact, more than 15 years ago, a similar (yet simpler) multiple RBE model was proposed by Jones et al for medulloblastoma patients [61]. Currently, there are an increasing number of ideas published in specialized journals and presented in international meetings on how to tackle the problem of proton radiobiological variability. In view of the difficulty to adopt variable RBE concepts clinically, some authors have proposed an optimization of RBE surrogates, such as LET alone, or LET*dose product. Care must be exercised with these solutions, as they contain implicit RBE models (expressed as the actual quantity to be optimized divided by the physical dose) that are not always easy to derive from their formulation and can lead to dimensional and numerical inconsistencies [16], unless compromise solutions (such as the one recently proposed by McMahon et al [62], with an LET-dependent dose weighting factor) are found. In this line, the MultiRBE approach can be combined with any other variable RBE model or doseweighting method deemed appropriate. For our implementation of the MultiRBE concept, the McNamara variable RBE model [28] was chosen as the most recent and developed of the so-called "empirical" RBE models based on in-vitro data. As the available body of in-vivo data grows [69], parameters of the variable RBE models can be adjusted and incorporated into the MultiRBE concept. In the end, every model will be subject to uncertainties related with the model parameters themselves, as well as with uncertainties in the alpha/beta ratios, dose and LET calculation, and so on. Since the physical dose coverage of the target is guaranteed with the MultiRBE concept, these uncertainties will affect only the redistribution of biological dose among organs at risk (OAR). However, this criticism applies to any estimation of biological dose, including the current standard of using a flat RBE of 1.1 as this model, simple as it is, is subject to uncertainties as well.

An obvious limitation of the MultiRBE approach is that it cannot, by definition, account for RBE differences within the PTV, as a fully-variable RBE model would do. Nevertheless, we strongly believe that the benefits of our model (ensuring PTV coverage in terms of physical dose) clearly outweigh this limitation, particularly as whatever healthy tissues exist within the PTV are deliberately irradiated with full prescription dose in order to ensure a correct treatment of the target.

The three cases presented aim to be representative of different situations from clinical practice, accounting for different doses per fraction (1.4-2 Gy), tumor alpha/beta values (1.4-10.5 Gy) and modulations $(2-12 \mathrm{~cm})$. The validity of the obtained results is, of course, dependent on the accuracy of the dose and LET calculation, which is based, respectively, on analytical and hybrid algorithms [43]. While discussing the accuracy of analytical dose calculations is outside the scope of this paper, we can remark that, thanks to the relatively smooth variation of RBE with LET, we are confident that local uncertainties in analytical calculation of LET do not produce significant variations in three-dimensional distributions of biological dose or biological dose-volume histograms [48], although, of course, the gold-standard of Monte Carlo calculation is always preferred [64].

The obtained results are also influenced by the choice of machine and treatment planning system (TPS): the presented data (reduction of biological dose and NTCPs) was obtained with a research TPS (matRad) using a 
generic proton machine: therefore, we can expect quantitative variations on the analyzed metrics depending on the specifics of the machine (different minimum and maximum range, spot sizes, etc.) and the choice of inverse planning program (cost function, implementation of restrictions, single-field or multi-field optimization, and so on). However, we would not expect a significant qualitative difference in the results, as the trends observed are caused by the MultiRBE concept and are therefore machine- and TPS- independent.

One consequence of using variable RBE models dependent on alpha/beta ratios is an abrupt variation in RBE maps at the edges of different organs. These will, in turn, cause abrupt edges in the biological dose distribution that might affect the optimization process. To solve this problem, additional blurring functions can be applied to the RBE models if smooth functions are required for the optimization.

Robust optimization has also been studied as a means to overcome the biological uncertainty in proton plans [65][21][68], not only because RBE uncertainties can be studied in independent scenarios, but also because robust techniques aiming at mitigating range uncertainties might also reduce the effect of a distal RBE increase. Therefore, the coupling of the MultiRBE concept with robust optimization techniques would be able to decrease LET values in the OARs adjacent to the target with a potential biological benefit of decreased toxicities.

The most important consequence of a potential implementation of the MultiRBE concept would be an improvement of proton treatment safety, reducing the probability of serious side effects, such as brainstem necrosis, with none or very limited ethical issues.

\section{Acknowledgements}

Work supported by the Spanish Government (FPA2015-65035-P, RTC-2015-3772-1), Comunidad de Madrid (S2013/MIT-3024 TOPUS-CM, B2017/BMD-3888 PRONTO-CM), European Regional Funds and the European Union's Horizon 2020 research and innovation programme under the Marie Sklodowska-Curie grant agreement No 793576 (CAPPERAM). This is a contribution for the Moncloa Campus of International Excellence, "Grupo de Física Nuclear-UCM", Ref. 910059. Part of the calculations of this work were performed in the "Clúster de Cálculo para Técnicas Físicas", funded in part by UCM and in part by EU Regional Funds.

The authors want to thank Mark Bangert and Hans-Peter Wieser for their support with matRad.

\section{References}

35 [1] ICRU 2007. Report 78: Prescribing, Recording, and Reporting Proton-Beam Therapy, International Commission on Radiation Units and Measurements, Bethesda, MD.

[2] Weyrather WK \& Kraft G 2004. RBE of carbon ions: experimental data and the strategy of RBE calculation for treatment planning. Radiotherapy and Oncology 73, S161.

40 function of biological endpoint, dose, and linear energy transfer. Phys Med Biol, 59 (22) R419-R472.

[4] Carabe, A., Moteabbed, M., Depauw, N., Schuemann, J., \& Paganetti, H. (2012). Range uncertainty in proton therapy due to variable biological effectiveness. Physics in Medicine \& Biology, 57(5), 1159.

[5] C. Grassberger \& H. Paganetti. Elevated LET components in clinical proton beams. Phys Med Biol, 56 (2011), pp. 6677-6691.

[6] H. Paganetti, 2017. Relating the proton relative biological effectiveness to tumor control and normal tissue complication probabilities assuming interpatient variability in alpha/beta Acta Oncol, 56, pp. 1379-1386.

[7] TS Underwood \& SJ McMahon, 2018. Proton Relative Biological Effectiveness (RBE): a Multi-scale Problem. British Journal of Radiology: 20180004.

[8] C.R. Peeler, D. Mirovic, U. Titt, et al.Clinical evidence of variable proton biological effectiveness in pediatric patients treated for ependymoma. Radiother Oncol, 121 (3) (2016), pp. 395-401

[9] Underwood, T. S. A., Grassberger, C., Bass, R., MacDonald, S. M., Meyersohn, N., Yeap, B. Y., ... \& Paganetti, H. (2018). Asymptomatic late-phase radiographic changes amongst chest wall patients are associated with a proton RBE exceeding 1.1. International Journal of Radiation Oncology• Biology• Physics.

[10] R.V. Sethi, D. Giantsoudi, M. Raiford, et al.Patterns of failure after proton therapy in medulloblastoma; linear energy transfer distributions and relative biological effectiveness associations for relapses. Int J Radiat Oncol 
Biol Phys, 88 (3) (2014), pp. 655-663

[11] D. Giantsoudi, J. Adams, S.M. MacDonald, et al.Proton treatment techniques for posterior fossa tumors: Consequences for LET and dose/volume parameters for the brainstem and organs at risk. Int J Radiat Oncol Biol Phys, 97 (2017), pp. 401-410.

5 [12] Merchant TE. Clinical controversies: proton therapy for pediatric tumors. Semin Radiat Oncol. 2013;23:97-108.

[13] D.J. Indelicato, S. Flampouri, R.L. Rotondo, et al.Incidence and dosimetric parameters of pediatric brainstem toxicity following proton therapy. Acta Oncol, 53 (10) (2014), pp. 1298-1304

[14] Ödén, J., DeLuca, P. M., \& Orton, C. G. (2018). The use of a constant RBE= 1.1 for proton radiotherapy is no longer appropriate. Medical physics, 45(2), 502-505.

10 [15] Willers, Henning, Antino Allen, David Grosshans, Stephen J. McMahon, Cläre von Neubeck, Claudia Wiese, and Bhadrasain Vikram. "Toward A variable RBE for proton beam therapy." Radiotherapy and Oncology (2018), in press.

[16] Jones, B. (2017). Clinical radiobiology of proton therapy: modeling of RBE. Acta Oncologica, 56(11), 13741378.

15 [17] H. Paganetti \& D. Giantsoudi, 2018. Relative Biological Effectiveness Uncertainties and Implications for Beam Arrangements and Dose Constraints in Proton Therapy. Seminars in Radiation Oncology 28 (3), 256.

[18] Mohan, R., Peeler, C. R., Guan, F., Bronk, L., Cao, W., \& Grosshans, D. R. (2017). Radiobiological issues in proton therapy. Acta Oncologica, 56(11), 1367-1373.

[19] Grassberger, C., \& Paganetti, H. (2017). Varying relative biological effectiveness in proton therapy: knowledge gaps versus clinical significance. Acta oncologica (Stockholm, Sweden), 56(6), 761-762.

[20] Sørensen, B. S., Bassler, N., Nielsen, S., Horsman, M. R., Grzanka, L., Spejlborg, H., ... \& Overgaard, J. (2017). Relative biological effectiveness (RBE) and distal edge effects of proton radiation on early damage in vivo. Acta Oncologica, 56(11), 1387-1391.

[21] Lühr, A., von Neubeck, C., Krause, M., \& Troost, E. G. (2018). Relative biological effectiveness in proton beam therapy-Current knowledge and future challenges. Clinical and translational radiation oncology.

[22] Jones, B., S. J. McMahon, and K. M. Prise. "The radiobiology of proton therapy: challenges and opportunities around relative biological effectiveness." Clinical Oncology 30, no. 5 (2018): 285-292.

[23] Ilicic, K., S. E. Combs, and T. E. Schmid. "New insights in the relative radiobiological effectiveness of proton irradiation." Radiation Oncology 13, no. 1 (2018): 6.

30 [24] Lühr, A et al, 2018. "Radiobiology of Proton Therapy": Results of an international expert workshop. Radiotherapy and Oncology.

[25] J.J. Wilkens \& U. Oelfke. A phenomenological model for the relative biological effectiveness in therapeutic proton beams. Phys Med Biol, 49 (13) (2004), pp. 2811-2825.

[26] Carabe-Fernandez, A., Dale, R. and Jones, B. (2007). The incorporation of the concept of minimum RBE (RBE$\mathrm{min}$ ) into the linear-quadratic model and the potential for improved radiobiological analysis of high-LET treatments. International Journal of Radiation Biology, 83(1), pp.27-39.

[27] M. Wedenberg, B.K. Lind, B. HardemarkA model for the relative biological effectiveness of protons: The tissue specific parameter alpha/beta of photons is a predictor for the sensitivity to LET changes. Acta Oncol, 52 (3) (2013), pp. 580-588.

40 [28] A.L. McNamara, J. Schuemann, H. PaganettiA phenomenological relative biological effectiveness (RBE) model for proton therapy based on all published in vitro cell survival data. Phys Med Biol, 60 (21) (2015), pp. 83998416.

[29] Elsasser T, Weyrather WK, Friedrich T, et al. Quantification of the relative biological effectiveness for ion beam radiotherapy: direct experimental comparison of proton and carbon ion beams and a novel approach for treatment planning. Int J Radiat Oncol Biol Phys. 2010;78:1177-1183.

[30] Friedrich T, Scholz U, Elsasser T, et al. Calculation of the biological effects of ion beams based on the microscopic spatial damage distribution pattern. Int J Radiat Biol. 2012;88:103-107.

[31] Hawkins RB. A microdosimetric-kinetic model of cell death from exposure to ionizing radiation of any LET, with experimental and clinical applications. Int J Radiat Biol. 1996;69:739-755.

50 [32] Okamoto H, Kanai T, Kase Y, et al. Relation between lineal energy distribution and relative biological effectiveness for photon beams according to the microdosimetric kinetic model. JRR. 2011;52:75-81.

[33] Frese, M. C., Wilkens, J. J., Huber, P. E., Jensen, A. D., Oelfke, U., \& Taheri-Kadkhoda, Z. (2011). Application of constant vs. variable relative biological effectiveness in treatment planning of intensity-modulated proton therapy. International Journal of Radiation Oncology* Biology* Physics, 79(1), 80-88.

55 [34] Underwood $\mathrm{T}$ et al, 2016. Can we advance proton therapy for prostate? Considering alternative beam angles and relative biological effectiveness variations when comparing against intensity modulated radiation therapy. Int $\mathrm{J}$ Radiat Oncol Biol Phys 95 (1):454-464, 2016.

[35] D. Giantsoudi, C. Grassberger, D. Craft, et al. LET-Guided Optimization in IMPT: Feasibility study and clinical potential. Int J Radiat Oncol Biol Phys, 87 (2013), pp. 216-222. 
[36] J. Unkelbach, P. Botas, D. Giantsoudi, et al.Reoptimization of intensity modulated proton therapy plans based on linear energy transfer. Int J Radiat Oncol Biol Phys, 96 (5) (2016), pp. 1097-1106.

[37] N. Bassler, J. Toftegaard, A. Lühr, et al.LET-painting increases tumor control probability in hypoxic tumors Acta Oncol, 53 (1) (2014), pp. 25-32.

5 [38] Cao, W., Khabazian, A., Yepes, P. P., Lim, G., Poenisch, F., Grosshans, D. R., \& Mohan, R. (2017). Linear energy transfer incorporated intensity modulated proton therapy optimization. Physics in Medicine \& Biology, 63(1), 015013.

[39] Sanchez-Parcerisa, D., \& Carabe-Fernandez, A. (2014). SU-E-T-555: A Protontherapy Inverse Treatment Planning System Prototype with Linear Energy Transfer (LET) Optimization. Medical Physics, 41(6Part20), 355.

10 [40] M. Fager, I. Toma-Dasu, M. Kirk, et al.Linear energy transfer painting with proton therapy: A means of reducing radiation doses with equivalent clinical effectiveness. Int J Radiat Oncol Biol Phys, 91 (5) (2015), pp. 10571064.

[41] Sanchez-Parcerisa, D., Kirk, M., Fager, M., Burgdorf, B., Stowe, M., Solberg, T., \& Carabe, A. (2016). Range optimization for mono-and bi-energetic proton modulated arc therapy with pencil beam scanning. Physics in Medicine \& Biology, 61(21), N565.

[42] Carabe-Fernandez, A., Kirk, M., Sanchez-Parcerisa, D., Fager, M., Burgdorf, B., Stowe, M., \& Solberg, T. (2015). SU-E-T-640: Proton Modulated Arc Therapy Using Scanned Pencil Beams. Medical physics, 42(6Part22), 3483-3483.

[43] Wieser, H. P., Cisternas, E., Wahl, N., Ulrich, S., Stadler, A., Mescher, H., ... \& Mairani, A. (2017). Development of the open-source dose calculation and optimization toolkit matRad. Medical physics, 44(6), 2556-2568.

[44] Bortfeld T. An analytical approximation of the Bragg curve for therapeutic proton beams. Med Phys. 1997;24:2024.

[45] Gottschalk B, Koehler AM, Schneider RJ, Sisterson JM, Wagner MS., Multiple Coulomb scattering of $160 \mathrm{MeV}$ protons. Nuclear Instrum Methods Phys Res B. 1993;74:467-490.

25 [46] Parodi K, Mairani A, Brons S, et al. Monte Carlo simulations to support start-up and treatment planning of scanned proton and carbon ion therapy at a synchrotron-based facility. Phys Med Biol. 2012;57:3759-3784.

[47] Sanchez-Parcerisa, D., Kondrla, M., Shaindlin, A., \& Carabe, A. (2014). FoCa: a modular treatment planning system for proton radiotherapy with research and educational purposes. Physics in Medicine \& Biology, 59(23), 7341.

30 [48] Sanchez-Parcerisa, D., Cortés-Giraldo, M. A., Dolney, D., Kondrla, M., Fager, M., \& Carabe, A. (2016). Analytical calculation of proton linear energy transfer in voxelized geometries including secondary protons. Physics in Medicine \& Biology, 61(4), 1705.

[49] Ezzell, G. A., Burmeister, J. W., Dogan, N., LoSasso, T. J., Mechalakos, J. G., Mihailidis, D., ... \& Shi, J. (2009). IMRT commissioning: multiple institution planning and dosimetry comparisons, a report from AAPM Task Group 119. Medical physics, 36(11), 5359-5373.

[50] David Craft, Mark Bangert, Troy Long, Dávid Papp, Jan Unkelbach; Shared data for intensity modulated radiation therapy (IMRT) optimization research: the CORT dataset, GigaScience, 3(1), 37.

[51] Miralbell, R., Roberts, S. A., Zubizarreta, E., \& Hendry, J. H. (2012). Dose-fractionation sensitivity of prostate cancer deduced from radiotherapy outcomes of 5,969 patients in seven international institutional datasets: $\alpha / \beta=$ 1.4 (0.9-2.2) Gy. International Journal of Radiation Oncology• Biology• Physics, 82(1), e17-e24.

[52] Emami, B., Lyman, J., Brown, A., Cola, L., Goitein, M., Munzenrider, J. E., ... \& Wesson, M. (1991). Tolerance of normal tissue to therapeutic irradiation. International Journal of Radiation Oncology* Biology* Physics, 21(1), 109-122.

[53] Stuschke, M., \& Thames, H. D. (1999). Fractionation sensitivities and dose-control relations of head and neck carcinomas: analysis of the randomized hyperfractionation trials. Radiotherapy and oncology, 51(2), 113-121.

[54] Withers, H. R., Peters, L. J., Taylor, J. M., Owen, J. B., Morrison, W. H., Schultheiss, T. E., ... \& Wang, C. C. (1995). Late normal tissue sequelae from radiation therapy for carcinoma of the tonsil: patterns of fractionation study of radiobiology. International Journal of Radiation Oncology• Biology• Physics, 33(3), 563-568.

[55] Liu, M., Moiseenko, V., Agranovich, A., Karvat, A., Kwan, W., Saleh, Z. H., ... \& Deasy, J. O. (2010). Normal tissue complication probability (NTCP) modeling of late rectal bleeding following external beam radiotherapy for prostate cancer: a test of the QUANTEC-recommended NTCP model. Acta Oncologica, 49(7), 1040-1044.

[56] Tucker, S. L., Dong, L., Bosch, W. R., Michalski, J., Winter, K., Lee, A. K., ... \& Mohan, R. (2007). Fit of a generalized Lyman normal-tissue complication probability (NTCP) model to grade $\geq 2$ late rectal toxicity data from patients treated on protocol RTOG 94-06. International Journal of Radiation Oncology* Biology* Physics, 69(3), S8-S9.

[57] Peeters, S. T., Hoogeman, M. S., Heemsbergen, W. D., Hart, A. A., Koper, P. C., \& Lebesque, J. V. (2006). Rectal bleeding, fecal incontinence, and high stool frequency after conformal radiotherapy for prostate cancer: normal tissue complication probability modeling. International Journal of Radiation Oncology• Biology• Physics, 66(1), 11-19. 
[58] Schaake, W., van der Schaaf, A., van Dijk, L. V., Bongaerts, A. H., van den Bergh, A. C., \& Langendijk, J. A. (2016). Normal tissue complication probability (NTCP) models for late rectal bleeding, stool frequency and fecal incontinence after radiotherapy in prostate cancer patients. Radiotherapy and Oncology, 119(3), 381-387.

[59] Semenenko, V. A., \& Li, X. A. (2008). Lyman-Kutcher-Burman NTCP model parameters for radiation pneumonitis and xerostomia based on combined analysis of published clinical data. Physics in Medicine \& Biology, 53(3), 737.

[60] Eisbruch, A., Ten Haken, R. K., Kim, H. M., Marsh, L. H., \& Ship, J. A. (1999). Dose, volume, and function relationships in parotid salivary glands following conformal and intensity-modulated irradiation of head and neck cancer. International Journal of Radiation Oncology• Biology• Physics, 45(3), 577-587.

10 [61] Jones, B., Wilson, P., Nagano, A., Fenwick, J., \& McKenna, G. (2012). Dilemmas concerning dose distribution and the influence of relative biological effect in proton beam therapy of medulloblastoma. The British journal of radiology, 85(1018), e912-e918.

[62] McMahon, S. J., Paganetti, H., \& Prise, K. M. (2018). LET-weighted doses effectively reduce biological variability in proton radiotherapy planning. Physics in Medicine \& Biology, 63(22), 225009.

[63] Paganetti, H., Niemierko, A., Ancukiewicz, M., Gerweck, L. E., Goitein, M., Loeffler, J. S., \& Suit, H. D. (2002). Relative biological effectiveness (RBE) values for proton beam therapy. International Journal of Radiation Oncology* Biology* Physics, 53(2), 407-421.

[64] Polster, L., Schuemann, J., Rinaldi, I., Burigo, L., McNamara, A. L., Stewart, R. D., ... \& Faddegon, B. (2015). Extension of TOPAS for the simulation of proton radiation effects considering molecular and cellular endpoints. Physics in Medicine \& Biology, 60(13), 5053.

[65] Ödén, J., Eriksson, K., \& Toma-Dasu, I. (2017). Incorporation of relative biological effectiveness uncertainties into proton plan robustness evaluation. Acta Oncologica, 56(6), 769-778.

[66] Sociedad Española de Oncología Radioterápica (2013). Manual Práctico de Oncología Radioterápica. 1 st ed. Madrid, Grupo Editorial abbvie, 201.

25 [67] Mendenhall, N. P., Li, Z., Hoppe, B. S., Marcus Jr, R. B., Mendenhall, W. M., Nichols, R. C., ... \& Henderson, R. (2012). Early outcomes from three prospective trials of image-guided proton therapy for prostate cancer. International Journal of Radiation Oncology* Biology* Physics, 82(1), 213-221.

[68] Giantsoudi, D., Unkelbach, J., Botas, P., Grassberger, C., \& Paganetti, H. (2017). Can Robust Optimization for Range Uncertainty in Proton Therapy Act as a Surrogate for Biological Optimization?. International Journal of Radiation Oncology• Biology• Physics, 99(2), S106-S107.

[69] Saager, M., Peschke, P., Brons, S., Debus, J., \& Karger, C. P. (2018). Determination of the proton RBE in the rat spinal cord: Is there an increase towards the end of the spread-out Bragg peak?. Radiotherapy and Oncology, 128(1), 115-120. 